\title{
A GENERALIZED GRONWALL-BELLMAN TYPE DELAY INTEGRAL INEQUALITY WITH TWO INDEPENDENT VARIABLES ON TIME SCALES
}

\begin{abstract}
YUZHEN MI
Abstract. Using a technique of monotonization, this paper investigates a generalized GronwallBellman type delay integral inequality with two independent variables on time scales. The result not only unifies some continuous inequalities and their discrete analogues but also extends some known integral inequalities on time scales. An application to the estimation of solutions of delay dynamic integral equations on time scales is also given.
\end{abstract}

Mathematics subject classification (2010): 26D15, 26D20.

Keywords and phrases: Integral inequality, delay, nonlinear, time scales.

\section{REFERENCES}

[1] S. HILGER, Analysis on measure chains - a unified approach to continuous and discrete calculus, Results Math. 18, 18-56 (1990).

[2] M. Bohner, A. Peterson, Dynamic Equations on time scales: An introduction with applications, Birkhauser, Boston, MA (2001).

[3] M. Bohner, A. Peterson, Advanced Dynamic Equations on time scales, Birkhauser, Boston, MA (2003).

[4] P. G. WANG, S. H. Tian, Y. H. Wu, Monotone iterative method for first-order functional difference equations with nonlinear boundary value conditions, Appl. Math. Comput. 203, 266-272 (2008).

[5] P. G. WANG, H. X. WU, Y. H. WU, Higher even-order convergence and coupled solutions for second-order boundary value problems on time scales, Comput. Math. Appl. 55, 1693-1705 (2008).

[6] R. P. Agrawal, S. F. Deng, W. N. Zhang, Generalization of a retard Gronwall-like inequality and its applications, Appl. Math. Comput. 165, 599-612 (2005).

[7] S. F. Deng, Nonlinear discrete inequalities with two variables and their applications, Appl. Math. Comput. 217, 2217-2225 (2010).

[8] D. B. PACHPATte, Inequalities for terminal value problems for differential equations, Tamkang J. Math. 33, 199-208 (2002).

[9] Y. Wu, X. P Li, S. F. Deng, Nonlinear delay discrete inequalities and their applications to Volterra type difference equations, Adv. Differ. Equ. Artcle ID 795145 (2010).

[10] Y. YAN, Nonlinear Gronwall-bellman type integral inequalities with maxima, Math. Inequal. Appli. 16 (3), 911-928 (2013).

[11] Q. H. MA, W. S. Cheung, Some new difference inequalities and their applications, J. Comput. Appl. Math. 202, 339-351 (2007).

[12] Y. Z. MI, Generalized integral inequalities for discontinuous functions with two independent variables and theri applications, J. Inequal. and Appl. 524 (2014).

[13] K. L. Zheng, Y. Wu, S. M. Zhong, Nonlinear Sum-difference Inequalities with Two Variables, Appl. Math. Comput. 1, 140-147 (2009).

[14] W. S. Cheung, Q. H. MA, On certain new Gronwall-OU-Iang type inntegral inequalities in two variables and their applications, J. Ineq. Appl. 4, 347-361 (2005).

[15] R. P. Agrawal, M. Bohner, A. Peterson, Inequalities on time scales: a survey, Math. Inequal Appl. 4, 5535-5557 (2001). 
[16] W. S. WANG, X. L. ZHOU, A generalized Pachpatte type inequality on time scales and application to a boundary value problem, Acta Math. Sci. (in chinese), 32A (92), 404-413 (2012).

[17] Q. H. MA, J. PECARIC, The bounds on the solutions of certian two-dimensional delay dynamil systems on time scales, Comput. Math. Appl. 61, 2158-2163 (2011).

[18] Q. H. FENG, B. BIN ZHENG, Generalized Gronwall-Bellman-type delay dynamic inequalities on time scales and their applications, Appl. Math. Comput. 218, 7880-7892 (2012).

[19] W. N. LI, Some Pachatte type inequalities on time scales, Computers. Math. Appl. 57, 275-282 (2009).

[20] T. L. WAng, R. Xu, Some integral inequalities in two independent variables on time scales, J. Math. Inequal. 6, 107-118 (2012).

[21] Q. H. Feng, F. W. Meng, Y. M. Zhang, J. C. Zhou, B. Zheng, Some delay integral inequalities on time scales and their applications in the theorey of dynamic equations, Abstract and applied analysis. 12, Article ID 538247, 21 pages (2012).

[22] E. A. Bohner, M. Boher, F. AKIn, Pachpatte Inequalities on time scales, J. Inequal. Pure. Appl. Math. 6 (1), 23-34 (2005).

[23] D. B. PachPatte, Explicit estimates on integral inequalities with time scale, J. Inequal. Pure Appl. Math. 7 (4), 1-8 (2006).

[24] W. N. LI, Some delay integral inequalities on time scales, Comput. Math. Appl. 59, 1929-1936 (2010).

[25] B. Zheng, Y. M. Zhang, Q. H. Feng, Some delay integral Inequalities in Two independent variables on time scales, J. Appl. Math. 1, Article ID 659563, 18 pages (2011). 